\title{
The utility of $A D C$ measurement techniques for differentiation of low- and high-grade clear cell RCC
}

\author{
Ahmet Aslan ${ }^{1 A, B, C, D, E, F}$, Ibrahim İnan ${ }^{1,2 A, B, D, E, F}$, Ahmet Aktan ${ }^{1 A, B, E, F}$, Ercan Ayaz ${ }^{1 A, B, E, F}$, Mine Aslan ${ }^{1 A, B, E, F}$, \\ Sıdıka Şeyma Özkanlı $3 A, B, D, E, F$, Asıf Yıldırım ${ }^{4 A, B, D, E}$, Ali Yıkılmaz ${ }^{1 A, B, D, E}$ \\ 'Department of Radiology, Istanbul Medeniyet University Göztepe Training and Research Hospital, Kadikoy, Istanbul, Turkey \\ ${ }^{2}$ Department of Radiology, Adiyaman University Training and Research Hospital, Adiyaman, Turkey \\ ${ }^{3}$ Department of Pathology, Istanbul Medeniyet University Göztepe Training and Research Hospital, Kadikoy, Istanbul, Turkey \\ ${ }^{4}$ Department of Urology, Istanbul Medeniyet University Göztepe Training and Research Hospital, Kadikoy, Istanbul, Turkey
}

\section{Abstract}

Purpose: To evaluate the diffusion properties of clear cell renal cell carcinoma (ccRCC) on magnetic resonance imaging (MRI) concerning their Fuhrman nuclear grades and sizes, and to compare the diagnostic performance of two ROI placement techniques for apparent diffusion coefficient (ADC) measurement (entire mass vs. only the darkest region of the mass).

Material and methods: Fifty-one ccRCC were enrolled in the study and grouped into low-grade ccRCC (Fuhrman grade 1 and 2,n=37) and high-grade ccRCC (Fuhrman grade 3 and 4, $n=14$ ). Selective ADC (Sel-ADC) measurement was performed by placing a circular ROI that included the darkest region of the tumour on ADC map images. Extensive ADC (Ext-ADC) measurement was performed by drawing an ROI that covered the entire tumour.

Results: The Sel-ADC value was lower in high-grade ccRCC $(p=0.019)$, whereas the Ext-ADC value did not show a statistically significant difference $(p=0.42)$. Sel-ADC value of a $\leq 1.405 \mathrm{~mm}^{2} / \mathrm{s}$ has a sensitivity, specificity, positive predictive value, negative predictive value, and accuracy value of $78.6,72.2,73.87,77.13$, and 75.4 , respectively, to differentiate high-grade from low-grade ccRCC. The size and Fuhrman grade of the ccRCC were inversely correlated with the Sel-ADC value; however, the correlations were weak $(r=-0.322, p=0.021$ and $r=-0.376, p=0.006$, respectively). There was no difference between ADC values of small $(\leq 4 \mathrm{~cm})$ and large $(>4 \mathrm{~cm})$ ccRCCs.

Conclusions: The ADC value of the darkest region in solid part of the ccRCC may play a role in predicting the nuclear grade of ccRCC.

Key words: magnetic resonance imaging, apparent diffusion coefficient, clear cell renal cell carcinoma, diffusion-weighted imaging, Fuhrman grading system.

\section{Introduction}

The radical or partial nephrectomy, percutaneous tumour ablation, or active surveillance are different treatment modalities for clear cell renal cell carcinoma (ccRCC) with optimal outcomes in selected patients [1-4]. Nevertheless, reliable treatment algorithms are not well established. The Fuhrman nuclear grade is a histopathological nuclear grading system for RCC, and also an independent factor for predicting the survival of the patient and the risk of metastasis [1-7].

Recent studies have focused on estimation of the ccRCC prognosis by using DWI features and Fuhrman

Correspondence address:

Ahmet Aslan, MD, Department of Radiology, Medical School of Istanbul Medeniyet University, 34722, Kadikoy, Istanbul, Turkey, e-mail: aslahmet@gmail.com Authors' contribution:

A Study design · B Data collection · C Statistical analysis · D Data interpretation · E Manuscript preparation · F Literature search · G Funds collection 
grades and a single region of interest (ROI) placed at the darkest portion on an apparent diffusion coefficient (ADC) map, or maximally enhancing part of the tumour to measure the ADC value $[1,3-6,8]$. However, a small sampling size may restrict the reproducibility of the DWI measurements because a single small ROI placement on ccRCC can display higher inter-observer variability and may not reflect the diffusion properties of a heterogeneous tumour appropriately $[4-6,8]$. In this study, we hypothesised that low-grade and high-grade ccRCCs could have different DWI properties, which may be helpful to foresee the tumour grade and aggressiveness preoperatively. We measured ADC values of ccRCCs by placing an ROI covering either the entire mass or only the darkest area on ADC map images. Furthermore, we analysed the correlation of ADC values with tumour size and Fuhrman nuclear grades.

\section{Material and methods}

The institutional review board committee approved the retrospective study design, and informed patient consent was waived.

\section{Patient selection}

Hospital information system was retrospectively reviewed for the keywords of "renal surgery", "partial nephrectomy", "radical nephrectomy", and "renal biopsy" from $1^{\text {st }}$ January 2012 to $1^{\text {st }}$ January 2016. A total of 158 patients who underwent renal intervention or surgery and had a histopathological diagnosis of various renal diseases were reviewed on a hospital information system. Among them, 35 patients were excluded due to having a renal parenchymal disease. Thirty-four patients were excluded because they had renal mass other than ccRCC such as papillary RCC $(n=10)$, chromophobe RCC $(n=5)$, transitional cell carcinoma $(n=2)$, oncocytoma $(n=7)$, angiomyolipoma $(n=8)$, and renal metastasis $(n=2)$. Eighty-nine patients with ccRCC were re-evaluated for the presence of convenient MRI for ADC measurement. Among them, 25 patients had no MRI within two months before surgery, five patients had no MRI with DWI, and 10 patients had MRI with intense motion artefacts, which hampered ADC measurements and excluded them from the study. A total of 51 ccRCCs in 49 patients constituted the final study group, which is summarised in Figure 1. Two patients had bilateral ccRCC, and rest of the selected patients had one ccRCC.

\section{Magnetic resonance imaging}

All MRI examinations were performed with a 1.5-T MRI scanner (General Electric, Signa HDxt 1.5 T GE Medical Healthcare, Milwaukee, WI, USA) by using a 16-channel phased array body coil before any intervention, surgery, or medical therapy. The MRI protocol included axial T1-weighted dual-echo and axial T2-weighted single-shot fast-spin echo sequences (SS-FSE), and DWI, followed by dynamic contrast-enhanced study (Figure 2).

Parameters for T1-weighted images were as follows: 140/2.1, 4.3 (TR/TE) ms; field of view (FOV), $400 \times 400 \mathrm{~mm}$; slice thickness, $6 \mathrm{~mm}$; slice gap, $1 \mathrm{~mm}$; matrix, $320 \times 192$; the number of excitations (NEX), 1. Parameters for T2-weighted SS-FSE were as follows: TR/TE, 890/90 ms; FOV, 400 $\times 400 \mathrm{~mm}$; slice thickness, $6 \mathrm{~mm}$; slice gap, $1 \mathrm{~mm}$; matrix, $256 \times 256$; NEX, 1.5. DWI was obtained before DCE-MRI with a breath-hold single-shot echo-planar SE sequence on the axial plane and tri-directional diffusion gradients. DWI parameters were as follows: TR/TE, 5400/65 ms; flip angle, $90^{\circ}$; FOV, $400 \times 400 \mathrm{~mm}$; matrix, $96 \times 128$; slice thickness, $6 \mathrm{~mm}$; slice gap, $1 \mathrm{~mm}$; NEX, 2. The gradient factors (b values) of 0 and $800 \mathrm{~s} / \mathrm{mm}^{2}$ were used, and ADC maps were created on a software-workstation system (Functool ver. 5x2.1.06, GE Medical Healthcare, Waukesha, Wisconsin, USA).

The dynamic contrast-enhanced (DCE) images (3D LAVA [20-35 s, 45-50 s, 90 s, and 180 s]) were obtained

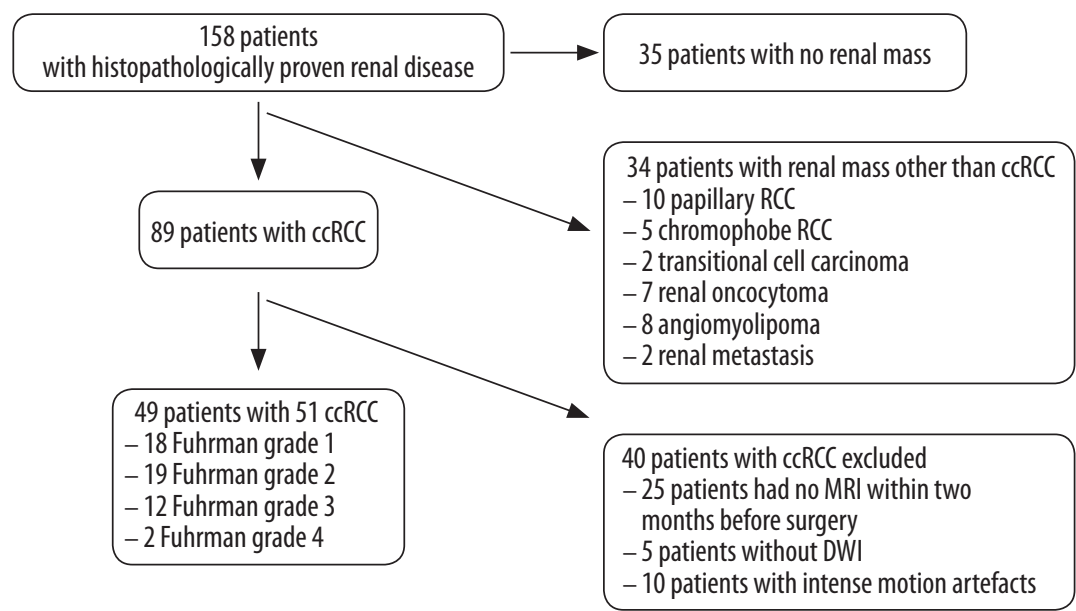

Figure 1. Flowchart of patient selection and study design 

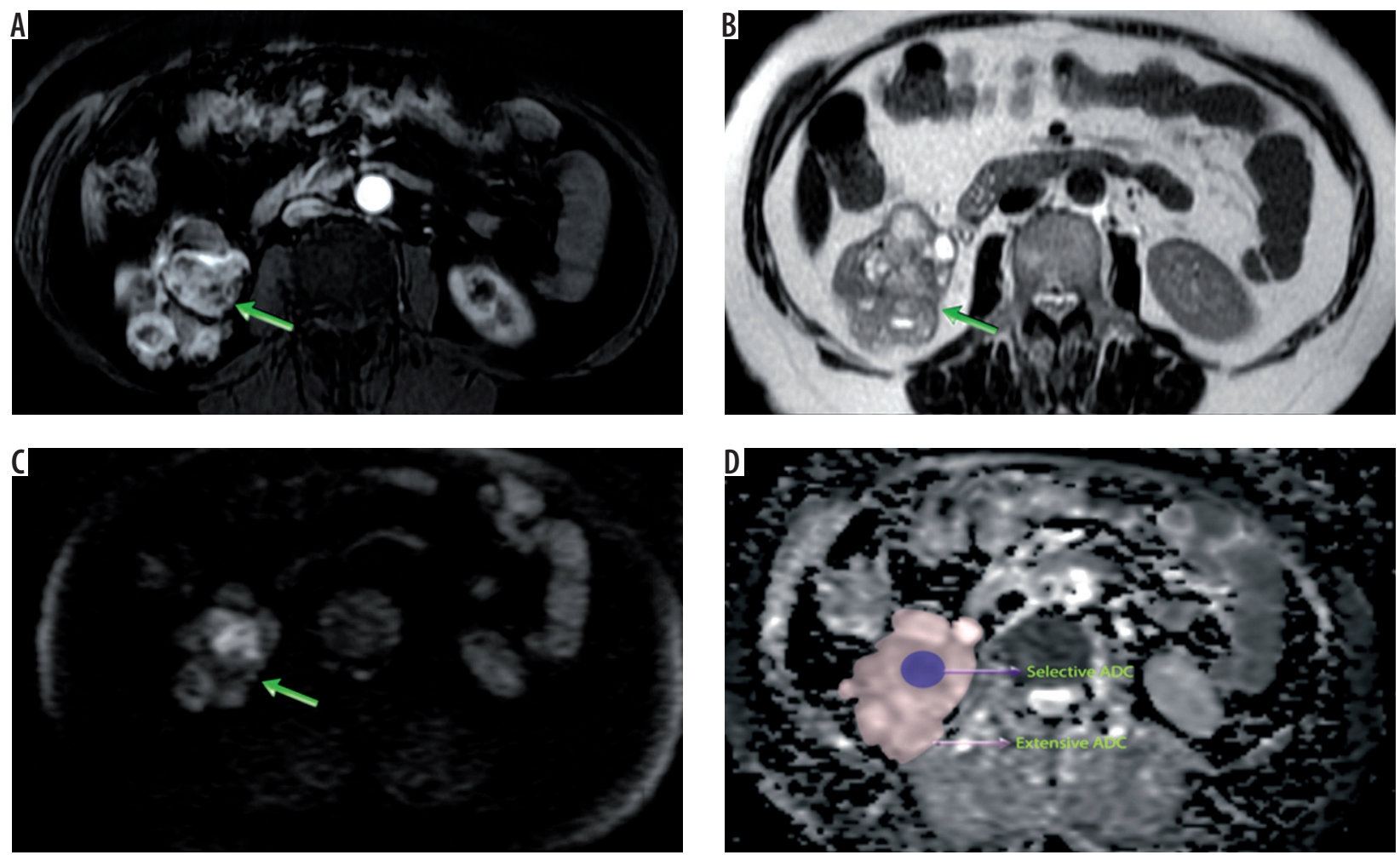

Figure 2. MRI abdomen of a 53-year-old female patient with ccRCC. Contrast-enhanced fat-saturated T1-weighted (A), T2-weighted (B), and diffusion-weighted images (C) demonstrate a predominantly cystic heterogeneous mass involving the lower pole of the right kidney (arrows). On an ADC map of the same patient (D), ROI replacements are shown

by administration of $0.1 \mathrm{mmol} / \mathrm{kg}$ of gadoteric acid (Dotarem, Guerbet, Roissy, France) with a saline flush of $20 \mathrm{cc}$. The parameters were as follows; TR/TE, 6.1/3.1 ms; flip angle, $12^{\circ}$; FOV $400 \times 320 \mathrm{~mm}$; slice thickness, $4.8 \mathrm{~mm}$; slice gap, $2.4 \mathrm{~mm}$; matrix, $288 \times 224$.

\section{Diagnostic criteria}

A pathologist with 12 years of experience in uropathology re-evaluated the histopathological specimens. The specimens were stained with haematoxylin-eosin, under a light microscope (Olympus BX51, Olympus Corporation, Tokyo 163-0914, Japan). The tumours from all patients were classified into four grades regarding the tumour nuclear size and shape, and chromatin pattern as described by the Fuhrman grading system regarding the highest Fuhrman grade $[1,2,9]$. After the cytological assessment, the tumours were merged into two groups as low (Fuhrman grade 1 and 2) and high (Fuhrman grade 3 and 4) grade ccRCC for analytical purposes $[10,11]$. Histopathological diagnosis and Fuhrman nuclear grading of the ccRCC served as the standard references for the statistical analysis.

\section{Image analysis}

Three radiologists with 4, 5, and 10 years of abdominal radiology experience evaluated the MRIs. The first observer collected the patients' demographic and histopathological data and evaluated the patients and their MRIs, as well as assessing the ccRCCs visually for the presence of cystic components. Two observers, who were blinded to the demographic and histopathological data of the patients, assessed the MRI on the MRI vendor's workstation in consensus. For the image evaluation, the size $(\mathrm{mm})$ was accepted as the mean of three maximum diameter measurement of the tumour on axial contrast-enhanced images on nephrographic phase. The selective ADC (Sel-ADC) $\left(\mathrm{mm}^{2} / \mathrm{s}\right)$ value was measured with a circular ROI of $0.5 \mathrm{~cm}^{2}$, including the corresponding darkest area on the ADC map. The extensive $\mathrm{ADC}\left(\right.$ Ext-ADC) value $\left(\mathrm{mm}^{2} / \mathrm{s}\right)$ was obtained by drawing an ROI involving the entire tumour on three different axial planes including the largest solid parts (Figure 2D). T1- and T2-weighted images, and DCE-MRI were used as references to depict the normal appearance of renal parenchyma and cystic/necrotic or haemorrhagic components of the tumour. The ADC measurements were performed, and the arithmetical mean of three ADC measurements was used for the statistical analysis.

\section{Statistical analysis}

The mean Sel-ADC and Ext-ADC values were compared between high-grade and low-grade ccRCCs. In addition, the correlations between Sel-ADC and Ext-ADC values with their size (small $[\leq 4 \mathrm{~cm}]$ and large $[>4 \mathrm{~cm}] \mathrm{RCC}$ ) and Fuhrman grades were assessed if there was a statistically significant difference. Continuous variables were assessed by the Shapiro-Wilks test for the normality of the 
Table 1. The size, $A D C$ values, and assessment of cystic component in different Fuhrman grades of ccRCCs

\begin{tabular}{|c|c|c|c|c|c|}
\hline \multirow[t]{2}{*}{ Fuhrman grades } & \multirow[t]{2}{*}{ Size (mm) } & \multicolumn{2}{|c|}{ ADC values $\left(\mathrm{mm}^{2} / \mathrm{s}\right)$} & \multirow{2}{*}{$\begin{array}{l}\text { Predominantly } \\
\text { cystic* } n(\%)\end{array}$} & \multirow{2}{*}{$\begin{array}{l}\text { Predominantly } \\
\text { solid } n(\%)\end{array}$} \\
\hline & & Sel-ADC & Ext-ADC ${ }^{a}$ & & \\
\hline Grade 1 & $53(20-154)^{\mathrm{a}}$ & $1.65(0.44-2.65)^{\mathrm{a}}$ & $2.09(1.06-2.83)^{\mathrm{a}}$ & $7(38.9 \%)$ & $11(61.1 \%)$ \\
\hline Grade 2 & $37(10-95)^{\mathrm{a}}$ & $1.53(0.84-2.18)^{\mathrm{a}}$ & $1.85(1.08-2.6)^{\mathrm{a}}$ & $3(15.8 \%)$ & $16(84.2 \%)$ \\
\hline Grade 3 & $45.5(27-107)^{\mathrm{a}}$ & $1.37(1.08-1.68)^{\mathrm{a}}$ & $1.90(1.39-2.38)^{\mathrm{a}}$ & $2(16.7 \%)$ & $10(83.3 \%)$ \\
\hline Grade 4 & $111.5(76-147)^{\mathrm{a}}$ & $1.07(0.73-1.4)^{\mathrm{a}}$ & $1.72(1.67-1.77)^{\mathrm{a}}$ & - & $2(100 \%)$ \\
\hline Low-grade & $40(10-154)^{\mathrm{a}}$ & $1.61(0.45-2.19)^{\mathrm{a}}$ & $1.95 \pm 0.4^{\mathrm{b}}$ & $10(27 \%)$ & $27(73)$ \\
\hline High-grade & $46(27-147)^{\mathrm{a}}$ & $1.37(0.74-1.68)^{\mathrm{a}}$ & $1.82 \pm 0.3^{b}$ & $2(14.3 \%)$ & $12(85.6 \%)$ \\
\hline Total & 51 & & & $12(23.5 \%)$ & $39(76.6 \%)$ \\
\hline
\end{tabular}

${ }^{a}$ median (range), ${ }^{b}$ mean $\pm S D, A D C$ - apparent diffusion coefficient, Sel-ADC - selective ADC, Ext-ADC - extensive ADC

*The masses were evaluated visually and grouped as predominantly cystic if they had more than $50 \%$ cystic component.

data and presented as mean $\pm \mathrm{SD}$ or median (range). The Mann-Whitney $U$ test or Student's $t$-test were performed to compare non-parametric and parametric variables, respectively. In addition, receiver operating characteristic (ROC) curve analysis was performed for each statistically significant parameter to establish an optimal cut-off value. Spearman's rank correlation analysis was used to show the correlations of size and Fuhrman grades of ccRCCs with Sel-ADC and Ext-ADC values. The correlations were accepted as very weak or no correlation if the coefficient value ( $\rho$ ) was $<0.4$, moderate if $r$ was 0.4-0.7, and good if $r$ was 0.8-1.0. All statistical analysis was made by using statistical software SPSS 22.0 (IBM Corporation, New York, United States). Statistical significance was deemed as $p<0.05$.

\section{Results}

Forty-nine patients (29 males [51.8\%] and 20 females [48.9\%]) with proper MRI were included in the study.

Table 2. $P$ values obtained from the comparison of Ext-ADC and Sel-ADC values, and size between different Fuhrman grades of cCRCCS

\begin{tabular}{|l|c|c|c|}
\hline Fuhrman grades & Sel-ADC & Ext-ADC & Size \\
\hline High, low-grade & $\mathbf{0 . 0 1 9 ^ { \mathrm { a } }}$ & $0.420^{\mathrm{b}}$ & $0.555^{\mathrm{a}}$ \\
\hline Grade 1-2 & $0.391^{\mathrm{a}}$ & $\mathbf{0 . 0 1 7 ^ { \mathrm { b } }}$ & $0.513^{\mathrm{a}}$ \\
\hline Grade 1-3 & $\mathbf{0 . 0 0 4 ^ { \mathrm { a } }}$ & $0.056^{\mathrm{b}}$ & $0.555^{\mathrm{a}}$ \\
\hline Grade 2-3 & $0.172^{\mathrm{a}}$ & $0.496^{\mathrm{b}}$ & $0.074^{\mathrm{a}}$ \\
\hline
\end{tabular}

aMann-Whitney $U$ test, ${ }^{\text {a }}$ Student's $t$-test

Statistically significant values are shown as bold characters.

CCRCC - clear cell renal cell carcinoma, Ext-ADC - extensive ADC, Sel-ADC - selective ADC

Table 3. Comparison of Sel-ADC and Ext-ADC values of small and large cCRCCs

\begin{tabular}{|l|c|c|c|c|}
\hline \multirow{2}{*}{} & \multicolumn{2}{|c|}{ Sel-ADC $^{\mathrm{a}}$} & \multicolumn{2}{c|}{ Ext-ADC $^{\mathrm{a}}$} \\
\cline { 2 - 5 } & Mean \pm SD & $p$ & Mean \pm SD & $p$ \\
\hline Small cCRCC $(n=22)$ & $1.58 \pm 0.31$ & $0.028^{\mathrm{b}}$ & $1.92 \pm 0.35$ & $0.944^{\mathrm{b}}$ \\
\hline Large ccRCC $(n=29)$ & $1.36 \pm 0.37$ & & $1.92 \pm 0.39$ & \\
\hline
\end{tabular}

${ }^{a} \mathrm{~mm}^{2} / \mathrm{s}$, bStudent's $t$-test

Sel-ADC - selective ADC, Ext-ADC - extensive ADC, $C C R C C$ - clear cell renal cell carcinoma, $S D$ - standard deviation
The mean age was $62.1 \pm 13.9$ years (range, 22 to 81 years). A total of 51 tumours were obtained (according to Fuhrman grading system; grade $1 n=18$ [35.3\%], grade $2 n=19$ [37.3\%], grade $3 n=12$ [23.5\%], and grade $4 n=2[3.9 \%])$ and grouped into low-grade ccRCC $(n=37,72.5 \%)$ and high-grade ccRCC $(n=14,27.5 \%)$ (Figure 1$)$. Twenty-three tumours involved the right kidney and 28 involved the left kidney. Histopathological data were obtained from partial nephrectomy in 23 patients and from total nephrectomy in 28 patients.

There was no statistically significant difference between the sizes of ccRCC regarding Fuhrman grades (Tables 1,2$)$. There were 21 small ccRCC $(\leq 4 \mathrm{~cm})$ (19 lowgrade and three high-grade ccRCCs) and 29 large ccRCCs $(>4 \mathrm{~cm})$ (18 low-grade and 11 high-grade ccRCCs). ExtADC was not significant between small and large ccRCCs, while Sel-ADC was statistically significant (Table 3 ). There were no correlations between small and large ccRCCs and their Fuhrman grades $(\rho=0.111, p=0.438)$. Also, there were no significant differences between Sel-ADC and Ext-ADC values of low- and high-grade ccRCCs regarding their size (small or large) (Table 4). The percentages of predominantly cystic ccRCCs were $38.9 \%$ in Fuhrman grade 1, 15.8\% in Fuhrman grade 2, and 16.7\% in Fuhrman grade 3 tumours. There were only two Fuhrman grade 4 ccRCCs and both were predominantly solid (Table 1). The mean Sel-ADC value was significantly lower in high-grade ccRCCs than in low-grade ccRCCs $(p=0.019)$. However, the mean Ext-ADC value difference between high-grade and low-grade RCCs was not significant $(p=0.42$ ) (Table 2). The mean Ext-ADC value was significantly higher in Fuhrman grade 1 tumours compared to Fuhrman grade $2(p=0.017)$. The mean Sel-ADC value was significantly lower in Fuhrman grade 3 than Fuhrman grade 1 ccRCCs $(p=0.004)$ (Table 2). The mean size of high and low-grade ccRCCs was not significant $(p>0.05)$ (Table 2). A ROC analysis was performed to obtain an optimal cut-off value for Sel-ADC value to differentiate low and high-grade ccRCC (area under the curve $=0.758, p<0.001)$. A Sel-ADC value of $\mathrm{a} \leq 1.405$ $\mathrm{mm}^{2} / \mathrm{s}$ has a sensitivity, specificity, positive predictive val- 
ue, negative predictive value, and accuracy value of 78.6, $72.2,73.87,77.13$, and 75.4 , respectively, to differentiate high-grade ccRCC from low-grade ccRCC.

The results of correlation analysis showed that Fuhrman grades of ccRCC correlated weakly and negatively with Ext-ADC and with Sel-ADC values (Table 5). Additionally, no correlation was found between tumour size and Fuhrman grade of $\operatorname{ccRCC}(\rho=0.113$ and $p=0.431)$. The size and Fuhrman grade of the ccRCC were inversely correlated with Sel-ADC value; however, the correlations were weak $(r=-0.322, p=0.021$ and $r=-0.376, p=0.006$, respectively).

\section{Discussion}

Clear cell RCC constitutes the greatest portion (70\%) of all renal cell carcinomas and is characterised by the worst prognosis and aggressive behaviour [1,3-6,9]. The main curative therapy option is radical nephrectomy. However, there are other options present, such as nephron-sparing surgery, minimally invasive methods (radiofrequency ablation, microwave ablation, or cryoablation techniques), or active surveillance, particularly in patients with comorbidities, bilateral RCCs, and high-risk patients for renal insufficiency $[3,6,7]$. A definitive treatment algorithm for ccRCCs has not been well-established yet. The stage, size, and nuclear grade of the ccRCC should be considered before starting the treatment. In this study, we compared two ROI placement techniques to measure ADC values of histopathologically proven and graded ccRCC according to the Fuhrman grading system, and we found a statistically significant difference between the low-grade and high-grade ccRCCs. Nonetheless, this difference was valid only for SelADC. Additionally, the ADC values of high- and low-grade ccRCC did not differ among small and large ccRCCs.

The Fuhrman nuclear grade plays a critical role in predicting the prognosis of patients with ccRCC. Unfortunately, it can only be evaluated on histopathologic specimens, and therefore it does not affect the management of the disease preoperatively [7,9]. The malignant masses demonstrate lower ADC values because of increased cellularity and narrowed extracellular spaces $[1,4,5,8,10]$. Thus, ADC values of ccRCCs, obtained from DWI may play an important role in predicting the aggressiveness and prognosis by anticipating the nuclear grade, and in predicting tumour prognosis before surgery [5]. Recent studies showed that the aggressiveness of ccRCC can be determined by DWI, and that ADC values were decreasing while the Fuhrman grades of ccRCCs were increasing $[1,4,6,8]$. Yu et al. and Rosenkrantz et al. found that highgrade ccRCCs had significantly lower ADC values than low-grade ccRCCs $[1,8]$. However, ADC values can be affected by various factors such as cellularity, type, nuclear grade, the proportion of cystic and solid components, haemorrhagic or proteinaceous cystic content of the ccRCC, and abscess. Therefore, ROI placement on ADC
Table 4. Comparison of ADC values of low- and high-grade cCRCCs among small and large ccRCCs

\begin{tabular}{|l|c|c|c|c|}
\hline \multirow{2}{*}{ Sel-ADC } & $\begin{array}{c}\text { Low-grade } \\
\text { ccRCC }\end{array}$ & $\begin{array}{c}\text { High-grade } \\
\text { (ccRCC }\end{array}$ & $p$ \\
\cline { 2 - 5 } & $\begin{array}{c}\text { Small ccRCC } \\
(n=22)\end{array}$ & $1.68(0.85-2.06)^{\mathrm{a}}$ & $1.37(0.26-1.61)^{\mathrm{a}}$ & $0.077^{\mathrm{b}}$ \\
\hline $\begin{array}{c}\text { Large cCRCC } \\
(n=29)\end{array}$ & $1.45(0.45-2.19)^{\mathrm{a}}$ & $1.37(0.74-1.68)^{\mathrm{a}}$ & $0.323^{\mathrm{b}}$ \\
\hline Ext-ADC & $\begin{array}{c}\text { Small cCRCC } \\
(n=22)\end{array}$ & $1.93(1.09-2.60)^{\mathrm{a}}$ & $1.75(1.63-2.28)^{\mathrm{a}}$ & $0.738^{\mathrm{b}}$ \\
\cline { 2 - 5 } & $\begin{array}{c}\text { Large cCRCC } \\
(n=29)\end{array}$ & $1.98(1.07-2.83)^{\mathrm{a}}$ & $1.87(1.40-2.38)^{\mathrm{a}}$ & $0.393^{\mathrm{b}}$ \\
\hline
\end{tabular}

${ }^{a}$ median (range) $\left(\mathrm{mm}^{2} / \mathrm{s}\right),{ }^{b}$ Mann Whitney $U$ test

Sel-ADC - selective ADC, Ext-ADC - extensive ADC, cCRCC - clear cell renal cell carcinoma

Table 5. Spearman's rank correlation analysis of ccRCCs between Fuhrman grades and size, Sel-ADC, and Ext-ADC values

\begin{tabular}{|l|c|c|} 
& Correlation coefficient $(\boldsymbol{\rho})$ & $p$ \\
\hline Size & 0.113 & 0.431 \\
\hline Ext-ADC & -0.335 & 0.016 \\
\hline Sel-ADC & -0.376 & 0.006 \\
\hline
\end{tabular}

Statistically significant values are shown as bold characters.

$C C R C C$ - clear cell renal cell carcinoma, Ext-ADC - extensive ADC, Sel-ADC - selective ADC

maps has gained importance because the non-homogeneous texture of ccRCC might affect the evaluation of DWI by hampering the accurate presentation of DWI $[5,8]$. In this study, we compared two different ADC measurements and found that Ext-ADC values were higher than the SelADC values in each Fuhrman grade. Similar to the literature, we found low ADC values in high-grade ccRCCs, and only the Sel-ADC value was statistically significant.

Heterogeneity of a renal mass generally indicates malignancy and predominantly ccRCC $[6,9,11]$. Necrosis and haemorrhage can cause heterogeneous texture and can be related to high-grade ccRCC. The fast growth of aggressive ccRCCs causes necrosis in the central part of the tumour due to insufficient vascular supply $[4,6]$. The cystic/necrotic or haemorrhagic portions of ccRCC might affect the evaluation of DWI by causing an increase in ADC values $[4,7]$. Zhang et al. used entire tumour-based ADC maps to compare histogram analysis of low- and high-grade ccRCC and found that low-grade ccRCC had significantly higher $\mathrm{ADC}$ values [6]. In our study, Ext-ADC was not significant in discriminating low-grade and high-grade ccRCCs, which may be related to the high percentage of cystic lowgrade ccRCCs contradictory to Zhang et al. study. In addition, we did not investigate histogram analysis to compare ADC data of the patients. The Sel-ADC and Ext-ADC values were inversely correlated with the Fuhrman grade of ccRCC in our study. Therefore, our findings suggest that the Sel-ADC value may represent the degree of aggressiveness rather than tumour size on MRI. However, the sensitivity and the specificity of Sel-ADC values are not 
high and should only be used as an ancillary finding to differentiate the low-grade and high-grade ccRCCs.

It is proposed that as the size of ccRCC grows, the grade of ccRCC also increases. Therefore, larger ccRCCs are expected to have lower ADC values. There are a limited number of studies focusing on the relation of the size and the grade of the ccRCC, and they mainly propose a significant correlation between the size and the nuclear grade of RCCs $[9,12,13]$. However, the mean size of ccRCCs did not differ and was inversely correlated among Fuhrman grades of ccRCC in our study. We also compared the ADC values of small and large ccRCCs regarding their nuclear grades. We found higher, statistically significant Sel-ADC values in small ccRCCs than in large ccRCCs. However, we could not differentiate low- and high-grade ccRCCs with their ADC values concerning the size of ccRCC (small or large). In a study by Zhang et al., ADC values of small RCCs were compared with benign renal masses, and they found higher ADC values for RCCs [13]. They found a value of $1.53 \pm 0.31\left(\times 10^{-3}\right.$ $\mathrm{mm}^{2} / \mathrm{s}$ ), similar to our Sel-ADC value on a $3.0 \mathrm{~T}$ MRI scanner. They proposed that ADC values can differentiate low- and high-grade RCCs among small RCCs. This discrepancy can be related to the morphology of the tumours, ROI placement on an ADC map that was unclear, and the MRI scanner (a 3.0. T MRI) [13].

There are some limitations to our study. Firstly, the retrospective design of the survey might have influenced the results. Secondly, there were only a small number of patients in the group of high-grade ccRCCs $(n=14)$, which degrades the reliability of the statistical results. Thirdly, interobserver and intraobserver reliability analysis can strengthen our results. We could add other RCC subtypes into the study. But we mainly focused on the ADC values of ccRCC. Also, the cellular structure of other subtypes of RCC may affect the role of ADC values in differentiating high- and low-grade RCC according to Fuhrman nuclear grading system. Finally, we did not perform histogram analysis in ADC measurement, which could also have provided more accurate results.

\section{Conclusions}

The size of ccRCC may have not a role in the determination of ccRCC aggressiveness. But ADC values of the darkest region of the ccRCC on ADC map may have a role in predicting the nuclear grade of ccRCC and should be used in conjunction with conventional MRI sequences as an ancillary finding. Optimal care should be given to ROI placement because the ADC values of the ccRCC may show variation and hamper the utility of DWI, depending on the internal structure of the tumour.

\section{Conflict of interest}

The authors report no conflicts of interest.

\section{References}

1. Rosenkrantz AB, Niver BE, Fitzgerald EF, et al. Utility of the apparent diffusion coefficient for distinguishing clear cell renal cell carcinoma of low and high nuclear grade. AJR Am J Roentgenol 2010; 195: W344-351.

2. Fuhrman SA, Lasky LC, Limas C. Prognostic significance of morphologic parameters in renal cell carcinoma. Am J Surg Pathol 1982; 6: 655-663.

3. http://www.auanet.org/guidelines/renal-mass-and-localized-renalcancer-new- (2017). (Accessed December 5th, 2017).

4. Maruyama M, Yoshizako T, Uchida K, et al. Comparison of utility of tumor size and apparent diffusion coefficient for differentiation of low- and high-grade clear-cell renal cell carcinoma. Acta Radiol 2015; 56: 250-256.

5. Goyal A, Sharma R, Bhalla AS, et al. Diffusion-weighted MRI in renal cell carcinoma: a surrogate marker for predicting nuclear grade and histological subtype. Acta Radiol 2012; 53: 349-358.

6. Zhang YD, Wu CJ, Wang Q, et al. Comparison of utility of histogram apparent diffusion coefficient and $\mathrm{R} 2{ }^{*}$ for differentiation of low-grade from high-grade clear cell renal cell carcinoma. AJR Am J Roentgenol 2015; 205: W193-201.

7. López-Costea MÁ, Bonet X, Pérez-Reggeti J, et al. Oncological outcomes and prognostic factors after nephron-sparing surgery in renal cell carcinoma. Int Urol Nephrol 2016; 48: 681-686.
8. Yu X, Lin M, Ouyang H, et al. Application of ADC measurement in characterization of renal cell carcinomas with different pathological types and grades by 3.0T diffusion-weighted MRI. Eur J Radiol 2012; 81: 3061-3066.

9. Reuter VE. The pathology of renal epithelial neoplasms. Semin Oncol 2006; 33: 534-543.

10. Wang H, Cheng L, Zhang X, et al. Renal cell carcinoma: diffusion-weighted MR imaging for subtype differentiation at 3.0 T. Radiology 2010; 257: 135-143.

11. Rothman J, Egleston B, Wong YN, et al. Histopathological characteristics of localized renal cell carcinoma correlate with tumor size: a SEER analysis. J Urol 2009; 181: 29-33; discussion 33-34.

12. Zhang $\mathrm{C}, \mathrm{Li} \mathrm{X}, \mathrm{Hao} \mathrm{H}$, et al. The correlation between size of renal cell carcinoma and its histopathological characteristics: a single center study of 1867 renal cell carcinoma cases. BJU Int 2012; 110 Pt B: E481-485.

13. Zhang HM, Wu YH, Gan Q, et al. Diagnostic utility of diffusion-weighted magnetic resonance imaging in differentiating small solid renal tumors $(\leq 4 \mathrm{~cm})$ at $3.0 \mathrm{~T}$ magnetic resonance imaging. Chin Med J (Engl) 2015; 128: 1444-1449. 